\title{
Rare Distal Anterior Choroidal Artery Aneurysm
}

\author{
Muhammad Zafrullah Arifin,, ${ }^{1}$ Julius July, ${ }^{2}$ Bilzardy Ferry, ${ }^{1}$ Ahmad Faried ${ }^{1}$ \\ ${ }^{1}$ Department of Neurosurgery, Faculty of Medicine, Universitas Padjdajaran-Dr. Hasan Sadikin General Hospital \\ ${ }^{2}$ Department of Neurosurgery, Faculty of Medicine, Pelita Harapan University-Siloam Hospital, Tangerang, \\ Indonesia
}

\begin{tabular}{|c|c|}
\hline \multirow[t]{3}{*}{ Abstract } & $\begin{array}{l}\text { Objective: To describe a rare patient with ruptur aneurysm case of distal } \\
\text { anterior choroidal artery (AChA) and intraventricular hemorrhage. A } 56 \text {-year } \\
\text { old female came to our hospital with chief complaint sudden onset of severe } \\
\text { headache and vomiting. }\end{array}$ \\
\hline & $\begin{array}{l}\text { Methods: Head computed tomography (CT)-scan and angiography on the } \\
\text { lesion was performed at the Department of Radiology, Siloam Hospital, } \\
\text { Tangerang, Indonesia. }\end{array}$ \\
\hline & $\begin{array}{l}\text { Results: Head CT-scan imaging revealed an intraventricular hemorrhage, } \\
\text { primarily in the right lateral ventricle, with slight enlargement of both lateral, } \\
3^{\text {rd }} \text { and } 4^{\text {th }} \text { ventricles. Angiography examination revealed a round vascular } \\
\text { lesion at the wall of the posterior cornu of the lateral ventricle and an } \\
\text { occlusion of the M1 base segment of the left middle cerebral artery. }\end{array}$ \\
\hline $\begin{array}{l}\text { Received: } \\
\text { June } 24,2015\end{array}$ & $\begin{array}{l}\text { Conclusions: The lesion, distal AChA aneurysm, at the posterior cornu } \\
\text { was reached using an infratemporal lobe approach with the help of } \\
\text { neuronavigation. Microsurgical clipping was successfully performed. }\end{array}$ \\
\hline $\begin{array}{l}\text { Revised: } \\
\text { April 24, } 2016\end{array}$ & $\begin{array}{l}\text { Keywords: Aneurysm, distal anterior choroidal artery, neuronavigation } \\
\text { [IJIHS. 2016;4(2):86-92] }\end{array}$ \\
\hline $\begin{array}{l}\text { Accepted: } \\
\text { August 3, } 2016\end{array}$ & pISSN: 2302-1381; eISSN: 2338-4506; http://dx.doi.org/10.15850/ijihs.v4n2.837 \\
\hline
\end{tabular}

\section{Introduction}

Reports on aneurysm of the distal anterior choroidal artery (AChA) are very rare. There are only 49 cases are reported in the literature. Twenty cases were associated with moyamoya disease; 10 cases with unknown causes; 8 cases are idiopathic; 3 cases were each associated with atherosclerosis and middle cerebral artery (MCA) occlusion; 2 cases with arteriovenous malformation (AVM), 1 case each for posterior cerebral artery (PCA) occlusion, cavernous angioma and head trauma (Table 1).

\section{Case}

A 56-year old female experienced a sudden onset of severe headache and vomiting. She Correspondence:

Bilizrdy Ferry, Department of Neurosurgery, Faculty of Medicine, Universitas Padjdajaran-Dr. Hasan Sadikin General Hospital

Jl. Pasteur No. 38, Bandung, Indonesia

e-mail: abilly_bfz@gmail.com was brought to a local hospital near her house and was hospitalized for 2 days. She was later referred to our hospital, the Siloam Hospital because her symptoms did not disappear. She still complained of severe headache but was fully alert without any neurological deficits. Results from chest x-ray, laboratory examinations and electrocardiogram were normal. Head CT-scan revealed that there was intraventricular hemorrhage with most of it was found in the right lateral ventricle; the left lateral, $3^{\text {rd }}$ and $4^{\text {th }}$ ventricles were slightly enlarged; and an arachnoid cyst in the magna cistern (sized $3 \times 2 \times 2 \mathrm{~cm}$ ). Angiography revealed the presence of a round, ruptured vascular lesion sized $5 \times 5 \mathrm{~mm}$ at the wall of posterior cornu of right lateral ventricle. Digital substraction angiography examination presented a small aneurysm at the right distal AChA and total obstruction at the left MCA, with collaterals observed. Occlusion of the M1 base segment of the left MCA and stenosis of the P2 segment of PCA were also found (Fig. 1 and 2). A repeat CT angiography was performed only to obtain same result, except 


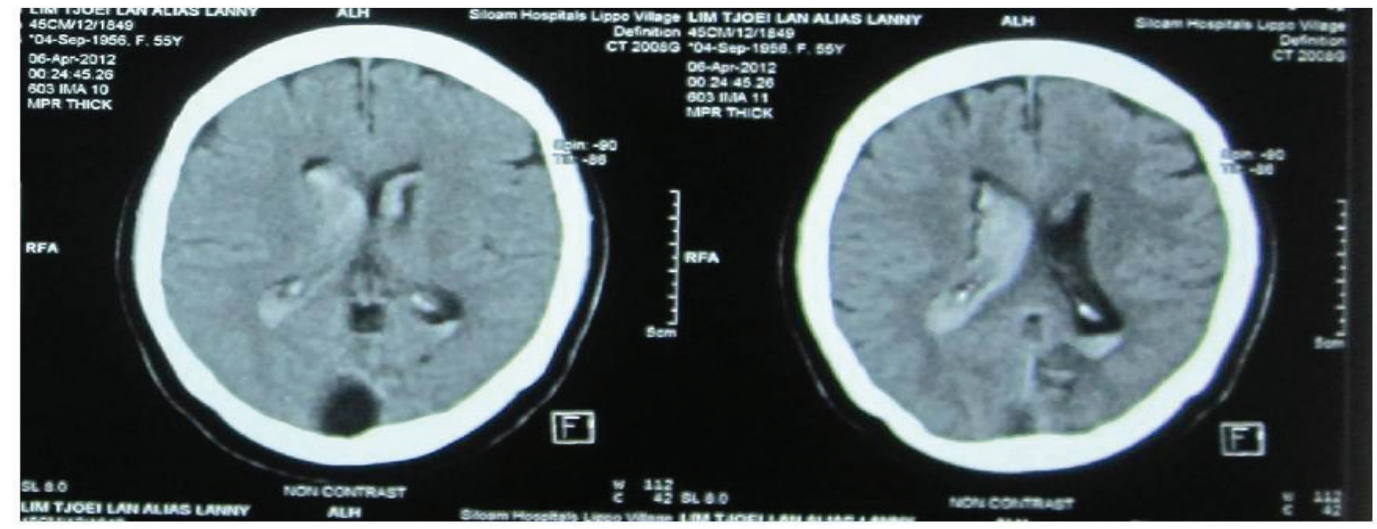

Fig. 1 Preoperative-axial Head CT-scan Showing an Intraventricular Hemorrhage on Both Side

decreasing density of the aneurysm, meaning that thrombosis had already occurred. The patient underwent surgery on the next day. A right temporooccipital craniotomy was conducted and the inferior temporal gyrus was accessed through neuronavigation. A saccular aneurysm with $5 \times 5 \mathrm{~mm}$ in the right lateral ventricle at the cornu posterior wall was found. The lesion was succesfully clipped, and an intraventricular drain was placed. One day after the operation, the patient's complaints were completely resolved and patient was discharged with no neurological deficit.

\section{Discussion}

Distal AChA aneurysm is a rare case. The first case of aneurysm of distal anterior choroidal artery was described by Strully in 1955. To the extend of our knowledge, there are only 50 cases have been found, including our case. ${ }^{1}$ The latest report for this case before our report is described by Oishi et al. ${ }^{2}$ in 2013. Shimizu et al. ${ }^{3}$ in 2013 reported a 6 years old (yo) patient, who was the youngest patient diagnosed with AVM while the oldest patient (84 yo) was reported by Nishida et al. ${ }^{4}$ in 2011.

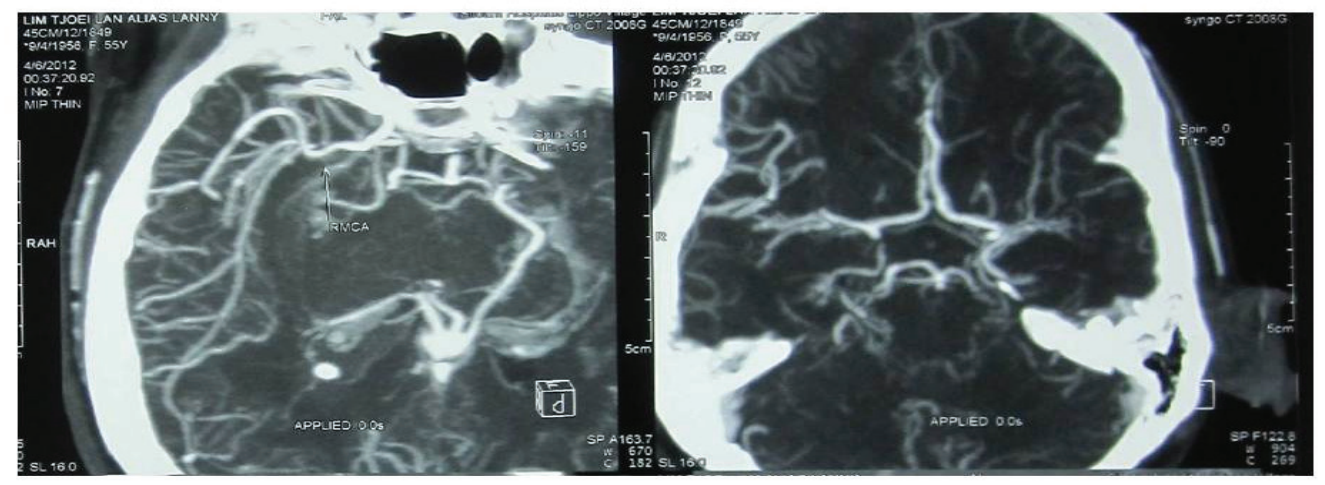

Fig. 2 Preoperative-axial CT-angiography Shows an Aneurysm at the Posterior Cornu Wall of Right Lateral Ventricle (with Diameter $\pm 5.6 \mathrm{~mm}$ ) 


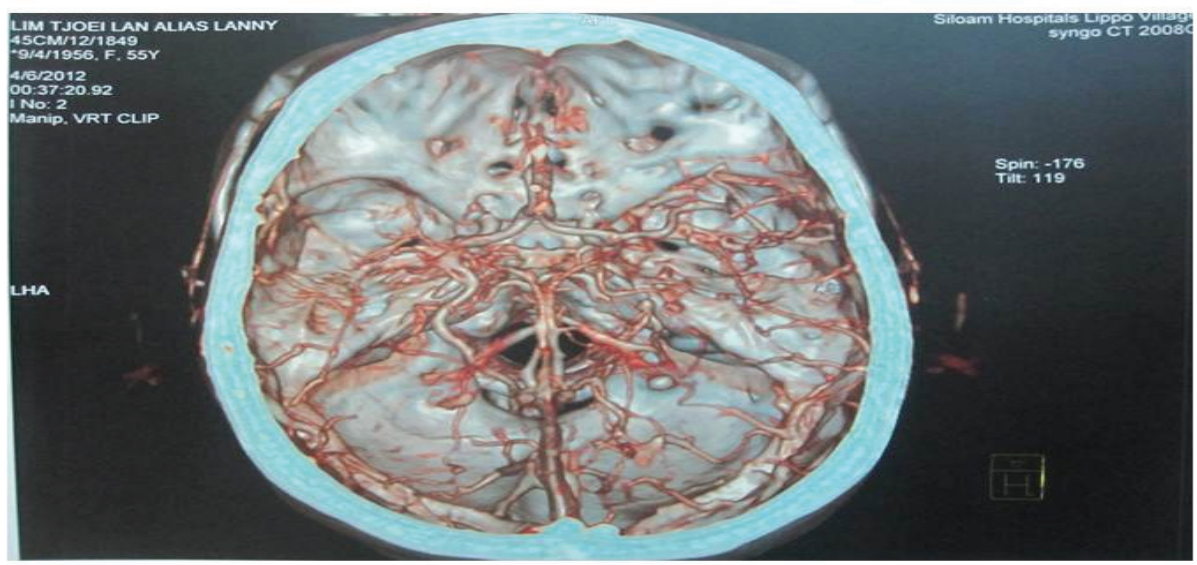

Fig. 3 Preoperative CT Angiography Showing an Aneurysm at the Distal Anterior Choroidal Artery
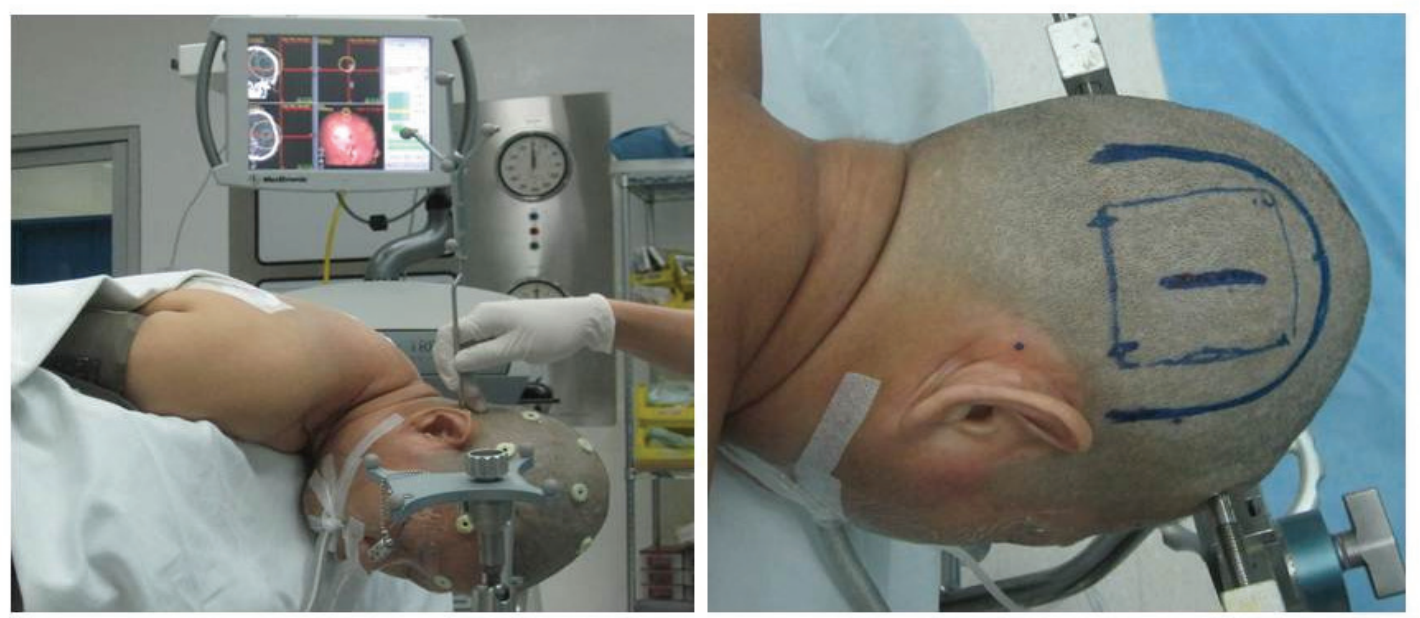

Fig. 4 Positioning of the Patient and Marking of the Operation Area for Neuronavigation

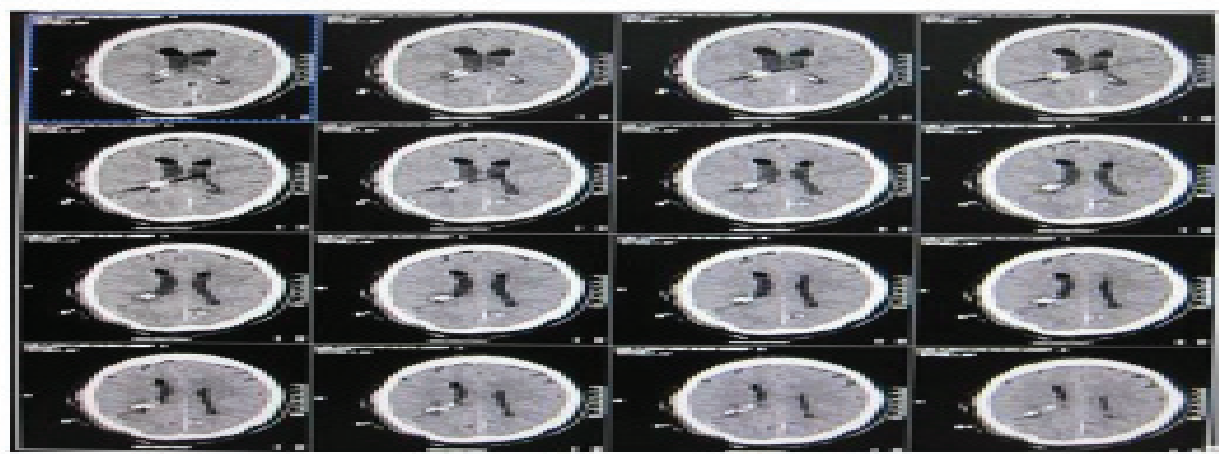

Fig. 5 Postoperative-axial Head CT-scan Showing an Intraventricular External Drainage Device and the Clip for AChA Aneurysm 
Table 1 List of Authors Reporting Patients with Distal Anterior Choroidal Artery Aneurysm

\begin{tabular}{|c|c|c|c|c|c|c|c|c|}
\hline No & Author & Location & Cause & Treatment & Outcome & $\begin{array}{c}\text { Age/ } \\
\text { Sex }\end{array}$ & $\begin{array}{c}\text { CT-scan } \\
\text { Result }\end{array}$ & $\begin{array}{c}\text { Pathologic } \\
\text { Result }\end{array}$ \\
\hline 1 & $\begin{array}{l}\text { Strully } \\
\text { (1955) }\end{array}$ & LTH & Idiopathic & Excision & $\begin{array}{c}\text { Severe } \\
\text { disability }\end{array}$ & $27 / F$ & NR & TGA \\
\hline 2 & $\begin{array}{l}\text { Caram et al. } \\
\text { (1960) }\end{array}$ & RBG & $\begin{array}{c}\text { Cavernous } \\
\text { angioma }\end{array}$ & NR & Death & $34 / \mathrm{M}$ & IVH & UTA \\
\hline 3 & $\begin{array}{l}\text { Cressman et } \\
\text { al. (1966) }\end{array}$ & LTH & Traumatic & $(-)$ & Death & $34 / \mathrm{M}$ & $\mathrm{ICH}$ & $(-)$ \\
\hline 4 & $\begin{array}{l}\text { Butler et al. } \\
\text { (1972) }\end{array}$ & Trigone & Unknown & Trapping & $\begin{array}{c}\text { Mild } \\
\text { disability }\end{array}$ & $15 / F$ & $\mathrm{ICH}+\mathrm{SAH}$ & NR \\
\hline 5 & $\begin{array}{l}\text { Papo et al. } \\
\text { (1973) }\end{array}$ & RTH & Atheros. & Resection & Death & $57 / \mathrm{M}$ & IVH & FA \\
\hline 6 & $\begin{array}{l}\text { Takeyama et } \\
\text { al. (1976) }\end{array}$ & $\begin{array}{l}\text { Left } \\
\text { trigone }\end{array}$ & Moyamoya & $(-)$ & Good & $43 / \mathrm{M}$ & SAH & $(-)$ \\
\hline 7 & $\begin{array}{l}\text { Tanaka et al. } \\
\text { (1978) }\end{array}$ & RTH & Moyamoya & $(-)$ & Death & $57 / F$ & IVH & $(-)$ \\
\hline 8 & $\begin{array}{l}\text { Takahashi et } \\
\text { al. (1980) }\end{array}$ & LTH & Moyamoya & NR & NR & $59 / \mathrm{M}$ & IVH & NR \\
\hline 9 & $\begin{array}{l}\text { Yamada et } \\
\text { al. (1981) }\end{array}$ & Right side & Moyamoya & NR & NR & $42 / F$ & $\mathrm{ICH}$ & NR \\
\hline 10 & $\begin{array}{l}\text { Furuse et al. } \\
\text { (1982) }\end{array}$ & $\mathrm{RBG}$ & Moyamoya & Resection & Good & $67 / M$ & $\mathrm{ICH}$ & FA \\
\hline 11 & $\begin{array}{l}\text { Kasamo et } \\
\text { al. (1984) }\end{array}$ & RTH & Moyamoya & $(-)$ & Death & $55 / F$ & SAH & $(-)$ \\
\hline \multirow[t]{3}{*}{12} & $\begin{array}{l}\text { Konishi et } \\
\text { al. (1985) }\end{array}$ & Right side & Moyamoya & $(-)$ & NR & $18 / F$ & IVH & SD \\
\hline & & Right side & Moyamoya & $(-)$ & Good & $13 / \mathrm{M}$ & IVH & SD \\
\hline & & Right side & Moyamoya & $(-)$ & Death & $34 / F$ & IVH & $(-)$ \\
\hline 13 & $\begin{array}{l}\text { Knuckey et } \\
\text { al. (1988) }\end{array}$ & LTH & Atheros. & Resection & Good & $46 / F$ & IVH & DAW \\
\hline 14 & $\begin{array}{l}\text { Sugiura et } \\
\text { al. (1988) }\end{array}$ & $\mathrm{RBG}$ & Moyamoya & Endov. & $\begin{array}{c}\text { Severe } \\
\text { disability }\end{array}$ & $47 / \mathrm{M}$ & IVH & $(-)$ \\
\hline 15 & $\begin{array}{l}\text { Onda et al. } \\
\text { (1988) }\end{array}$ & $\begin{array}{l}\text { Left } \\
\text { trigone }\end{array}$ & Moyamoya & $(-)$ & Good & $43 / M$ & SAH & SD \\
\hline 16 & $\begin{array}{l}\text { Inagawa et } \\
\text { al. (1990) }\end{array}$ & LTH & Idiopathic & $(-)$ & Death & $75 / F$ & $\mathrm{IVH}+\mathrm{SAH}$ & $\mathrm{TA}$ \\
\hline 17 & $\begin{array}{l}\text { Nakai et al. } \\
\text { (1992) }\end{array}$ & $\begin{array}{l}\text { Right } \\
\text { trigone }\end{array}$ & Moyamoya & Resection & $\begin{array}{c}\text { Mild } \\
\text { disability }\end{array}$ & $42 / \mathrm{M}$ & $\mathrm{ICH}$ & $\mathrm{TA}$ \\
\hline 18 & $\begin{array}{l}\text { Nishihara et } \\
\text { al. (1993) }\end{array}$ & RTH & Idiopathic & Resection & Good & $34 / F$ & IVH & $\mathrm{TA}$ \\
\hline 19 & $\begin{array}{l}\text { Hamada et } \\
\text { al. (1994) }\end{array}$ & $\begin{array}{c}\text { Lateral } \\
\text { ventricle }\end{array}$ & Moyamoya & Trapping & Good & $48 / \mathrm{F}$ & IVH & NR \\
\hline 20 & $\begin{array}{l}\text { Hung et al. } \\
\text { (1996) }\end{array}$ & $\begin{array}{l}\text { Cisternal } \\
\text { segment }\end{array}$ & Idiopathic & Trapping & Good & $35 / F$ & SAH & NR \\
\hline
\end{tabular}


21 Morgenstern Temporal et al. (1996) horn Idiopathic

Kawai et al.

22 (1997)

23 Yoneoka $e t$ al. (1998)

24 Yanaka et al. (2000)

Matsuura et al. (2000)

26 Lee et al. (2001)

27 Kuroda et al. (2001)

28 Wong et al. (2003)

29 Ali et al. (2004)

30 Nishio et al. (2004)

31 Ahn et al. (2006)

32 Inci et al.

3 Gandhi et al. (2008)

34 Yurt et al. (2009)

35 Kim et al.

35 (2009)

36 Yang et al.

37 Choulakian et al. (2010)

38 Nishida et al. (2011)

39 Leveque et al. (2011)

40 Dolati et al. (2012)

41 He et al. (2013)
RTH Moyamoya

Right side

Right side

Cisternal segment

Right trigone

Unknown AVM

Idiopathic

Moyamoya

NR Unknown

Resection

Rev.

Temporal horn

Moyamoya

Clipping

NR Unknown

Clipping

NR

Unknown

Embolization

$(-)$

Left side Unknown

Temporal

Idiopathic

Resection

Temporal Idiopathic

Resection horn

\begin{abstract}
NR
\end{abstract}
Unknown

Right

side

Unknown

Clipping

Clipping

Vegetative

NR Moyamoya

NR Moyamoya

Endov.

NR Moyamoya

Endov.

NR Moyamoya

Endov.

MCA

RTH

Left side

Occlusion

Endov.

Endov.

Endov.

NR $\begin{gathered}\text { PCA } \\ \text { Occlusion }\end{gathered}$

Left side Unknown

Clipping

Right side
Unknown
Conservative

$60 / \mathrm{F}$

Good

19/F $\quad \mathrm{ICH}+\mathrm{IVH}$

NR

Death

$37 / \mathrm{F} \quad \mathrm{ICH}+\mathrm{SAH}$

NR

NR

M

SAH

NP

Good

NR

$\mathrm{ICH}+\mathrm{IVH}$

NP

NR

$43 / \mathrm{F}$

IVH

NR

Good 56/F IVH

NP

Good 38/F IVH

NP

Good

NR

IVH

NP

Mild disability

84/F IVH

NP

Good 50/F

IVH

NP

Good

55/M

IVH

NP

Good

M

IVH

NP

Good

M

IVH

NP 
Muhammad Zafrullah Arifin, Julius July, et al.

\begin{tabular}{|c|c|c|c|c|c|c|c|c|}
\hline 42 & $\begin{array}{l}\text { Shimizu et } \\
\text { al. (2013) }\end{array}$ & $\begin{array}{c}\text { Lateral } \\
\text { posterior }\end{array}$ & Atheros. & Endov. & Good & $43 / F$ & $\mathrm{ICH}$ & $\mathrm{NP}$ \\
\hline & & NR & AVM & Endov. & Good & $6 / F$ & $\mathrm{ICH}$ & $\mathrm{NP}$ \\
\hline 43 & $\begin{array}{l}\text { Oishi et al. } \\
\text { (2013) }\end{array}$ & RTL & $\begin{array}{c}\text { MCA } \\
\text { occlusion }\end{array}$ & Endov. & $\begin{array}{c}\text { Mild } \\
\text { disability }\end{array}$ & $75 / F$ & ICH & NP \\
\hline 44 & $\begin{array}{l}\text { Our case } \\
(2014)\end{array}$ & RTH & $\begin{array}{c}\text { MCA } \\
\text { occlusion }\end{array}$ & Clipping & Good & $56 / F$ & IVH & $\mathrm{NP}$ \\
\hline
\end{tabular}

Notes:

Atheros.: Atherosclerotic

DAW: Degenerated artery wall

Endov.: Endovascularization

FA: False aneurysm

NR: Not reported

NP: Not performed

ICH: Intracerebral haemorrhage

IVH: Intraventricular haemorrhage

LTH: Left temporal horn

RBG: Right basal ganglia

RTH: Right temporal horn

RTL: Right temporal lobe

Rev.: Revascularization

SAH: Subarachnoid haemorrhage

SD: Spontaneus disappearance

TGA: Thrombosed giant aneurysm

TA: True aneurysm

UTA: Unruptured true aneurysm

No differences between gender were found. In terms of cause, most of the cases, i.e. 20 cases , were caused by moya-moya disease. ${ }^{5}$

In the case presented here, on the opposite side from thelocation of aneurysm, an occlusion of M1 segment of left MCA and narrowing or stenotic of P2 segment of left PCA were found; hence, it can be concluded that the cause of the aneurysm was MCA occlusion. In this case, the location of aneurysm is on the right lateral intraventricular at the temporal horn, known as plexal segment, while almost all patients had the aneurysm located in temporal horn (Table 1) eventhough most authors did not mention the location of the aneurysm in their case report. In this report, our patient was discharged from the hospital uneventfully. It is so unfortunate that most studies did not mention the outcome of their case.

Direct micosurgical intervention through

\section{References}

1. Strully KJ. Successful removal of intraventricular aneurysm of the choroidal artery. J Neurosurg. 1955;12(3):317-21.

2. Oishi H, Suga Y, Nonaka S. Endovascular therapy of ruptured distal anterior choroidal artery aneurysm associated with moyamoya pattern collateralization secondary to middle cerebral artery occlusion. Indian J Neurosurg. 2013;2(3):278-81.

3. Shimizu T, Naito I, Fujita K, Miyamoto N, Sato $\mathrm{K}$, Aishima $\mathrm{K}$, et al. Aneurysm of ruptured distal chroroidal artery embolized using n-butyl cyanoacrylate: case report. JNET. a transtemporal or ventricular approach is one of the options for managing distal AchA aneurysm with somehow additional damage to the brain and its collateral circulation may not be avoidable. ${ }^{6}$ Several reported cases of the distal AChA show that the cases were successfully treated using coils and n-butyl cyanoacrylate (nBCA) liquid embolization, arguing that the endovascular technique is a promising modality for this rare case..$^{2-9}$

In conclusion, in the present case, aneurysm of distal choroidal anterior artery can be managed. Timing of surgery with great caution may be an advantage to improve the prognosis of the patient. The choice of treatment depends on the available expertise and equipment; the latest report has argued that the endovascular technique is a promising modality for this rare case. 
artery aneurysm. Surg Neurol. 2007;67(1):4652.

7. Yang S, Yu JL, Wang HL, Wang B, Lou Q. Endovascular embolization of distal anterior choroidal artery aneurysm associated with moyamoya disease: a report of two cases and a literature review. Interv Neuroradiol. 2010;16(5):433-41.

8. Leveque $\mathrm{M}$, McLaughlin $\mathrm{N}$, Laroche $\mathrm{M}$,
Bojanowski MW. Endoscopic treatment of distal choroidal artery aneurysm: Case report. J Neurosurg. 2011;114(1):116-9.

9. Choulakian A, Drazin D, Alexander MJ. NBCA embolization of a ruptured intreventricular distal anterior choroidal artery aneurysm in a patient with moyamoya disease. J Neurointervent Surg. 2010;2(4):368-70. 